\title{
THE NON-LINEAR PROPERTIES OF INCOMMENSURATE PHASES IN THE SYSTEMS WITH SCALAR ORDER PARAMETER
}

\author{
S. V. Berezovsky, V. F. Klepikov, Yu. V. Sereda \\ Scientific and Technological Center of Electrophysics, \\ National Academy of Sciences of Ukraine, \\ 28 Chernyshevs'kyi Str., P. O. BOX 8812, Kharkiv, UA-310002, Ukraine \\ (Received January 5, 1998; received in final form March 17, 1998)
}

\begin{abstract}
Non-linear properties of the order parameter distributions in the systems with one-component order parameter are considered. It is shown that the solutions expressed through Jacobi elliptic functions give more information about the behaviour of such a system in comparison with the models in which the order parameter distribution is approximated with the harmonic series. The developed approach provides a new mechanism of system characteristics dependence on the external parameters.
\end{abstract}

Key words: Landau theory of phase transitions, order parameter, spatial distribution, Jacobi elliptic functions.

PACS numbers: 64.60.My; 64.70.Kb; 05.70.Fh

\section{INTRODUCTION}

The incommensurate states are known to exist in many materials [1]. Their appearance is caused different reasons. The properties of modulated phases are different as well. Following the classification proposed in [2] we point out the systems of type I and type II.

In the case of type I system the order parameter has two (or more) components $\eta(x)$ and $\xi(x)$, and the invariant linear on the gradients can be constructed (the Lifshits invariant $[\eta(x) \cdot \partial \xi(x) / \partial x-\xi(x) \cdot \partial \eta(x) / \partial x])$. Close to the point of transition from the normal phase into the incommensurate state the order parameter spatial distribution is sinusoidal. But it distorts to the step-like dependence as the temperature is lowered. The transition between modulated and commensurate phases can be of the second order (at least in the samples without defects).

In the case of type II systems the order parameter $\varphi(x)$ transforms under one-dimensional irreducible representation of the symmetry group of disordered phase. The Lifshits invariant is forbidden. The order parameter spatial distribution is sinusoidal almost in all the temperature region of the existence of the incommensurate phase. The phase transition into the commensurate phase is always of the first order.

The differences of type II systems, especially their specific non-linear properties [3], are discussed in many papers. In particular, the numerical analysis in the framework of multiplane approximation [4] shows that the contribution of higher harmonics in the order parameter distribution increases when the $g \cdot \varphi^{2}(x)[\partial \varphi(x) / \partial x]^{2}$ invariant with large enough coefficient $g$ is taken into account in the thermodynamical potential expression. Moreover, the $g$-term mechanism permits to describe the order parameter dependence on temperature, pressure, electric fields correctly [4]-[6].

At the same time, in the theory of systems with scalar order parameter the solutions analogous to the multi- soliton distributions known for the type I systems [7] are not found. The one-harmonic approximation is often used to describe the spatial dependence of the order parameter.

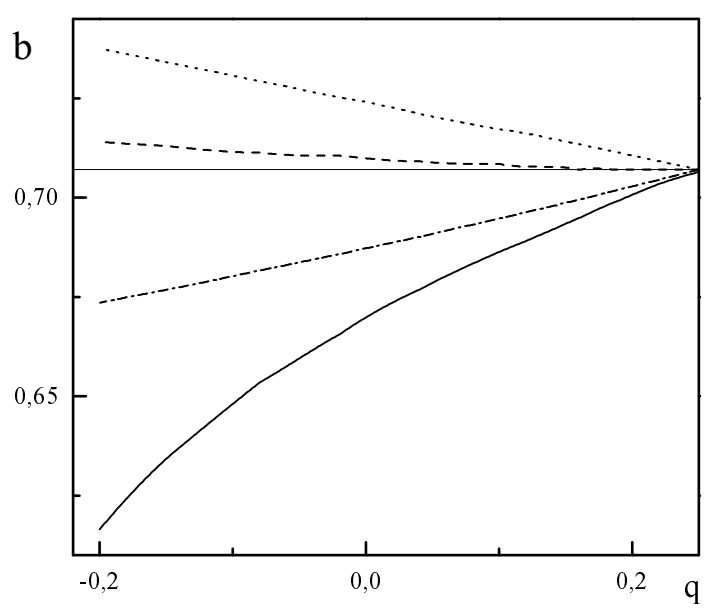

Fig. 1. The dependence of wave number $b$ on the effective temperature $q$ in model (1), (9). The solid curve shows $b(q)$ for the case $g=-10$, the dashed curve is for $g=-1$, the dotted curve corresponds to the case $g=+0.1$. The dashed-dotted curve is calculated according to the (3), (4), $g=-1$. The solid line corresponds to the value $b_{0}^{2}(q)=0.5$ (the case $g=0$ in equation (4))

In $[8,9]$ within the framework of Landau-type theory the approach is developed which allows to obtain new non-linear solutions for the scalar order parameter distributions in the incommensurate phase. In the 
present paper we continue investigating the properties of the found solutions.

In the section II we formulate the problem and point out the class of considered solutions. These distributions are expressed through the Jacobi elliptic sinus the parameters of which are defined by means of the minimization of the system thermodynamical potential.

In section III the results of numerical investigations of the solution parameters and the phase transition characteristics are presented.

Section IV contains discussion and conclusions. The order parameter distributions under consideration correctly describe the known properties of the type II systems. But they give more information in comparison with multi-plane approximation because they allow to understand the non-linear behaviour of one-component order parameter systems in more detail and provide a new mechanism for describing the order parameter dependence on the external parameters. The $g$-term can, in principle, provide the existence of multi-soliton states in the type II systems. But the growth of the absolute value of parameter $g$ simultaneously leads to an essential narrowing of the temperature interval where the incommensurate phase exists and makes the observation of such states a difficult task.

\section{THE Sn-DISTRIBUTIONS OF SCALAR ORDER PARAMETER}

Phase transitions in the system with one-component order parameter can be described phenomenologically on the basis of the following thermodynamical potential [4, $5,9]$ :

$$
\begin{aligned}
\Phi & =\int_{0}^{L}\left[\left(\varphi^{\prime \prime}\right)^{2}-g\left(\varphi \varphi^{\prime}\right)^{2}-\gamma\left(\varphi^{\prime}\right)^{2}\right. \\
& \left.+g \varphi^{2}+\frac{p}{2} \varphi^{4}+\frac{1}{3} \varphi^{6}\right] d x
\end{aligned}
$$

where $\varphi(x)$ is the order parameter, $L$ is the crystal length along the $x$-axis (the direction of modulation in the incommensurate phase), $\varphi^{\prime} \equiv \partial \varphi(x) / \partial x ; g, \gamma, g, p$, are the material parameters. In expression (1) all the parameters, the order parameter and the coordinate $x$ are dimensionless [10]. We suggest that the temperature is the only external parameter causing the phase transformations in the system, and $q=q \cdot\left(t-t_{0}\right)$, while other parameters do not depend on the temperature.

The variational differential equation corresponding to functional (1) is given by

$$
\begin{aligned}
& \varphi^{(I V)}+g\left(\varphi^{2} \varphi^{\prime \prime}+\varphi \varphi^{\prime 2}\right)+\gamma \varphi^{\prime \prime} \\
& +q \varphi+p \varphi^{3}+\varphi^{5}=0 .
\end{aligned}
$$

Due to taking into account of the gradient terms the thermodynamical potential (1) allows to describe not only homogeneous phases such as disordered and commensurate ones but incommensurate phase as well [3]. The $g$-term permits to explain the order parameter dependence on the external parameters [4-6]. In particular, if the order parameter spatial distribution is considered in the plane wave approximation [11]

$$
\varphi(x)=a \cdot \sin (b x)
$$

then the $g$-term provides the proper modulation period dependence on the temperature. Really, the minimization of functional (1) in respect to the parameters $a, b$ in the case when the order parameter is expressed according to (3) gives [4]:

$$
b^{2}=\frac{\gamma}{2}+\frac{1}{8} g a^{2}
$$

If $g<0$ and the temperature is lowered the order parameter amplitude $a$ increases [4] that leads to simultaneous decreasing of the wave number $b$. As a consequence, the modulation period $T=2 \pi / b$ grows under the mentioned conditions. Such a behaviour qualitatively corresponds to the experimental data for ferroelectrics (for example, thiourea and sodium nitrite), some magnetics etc. [1].

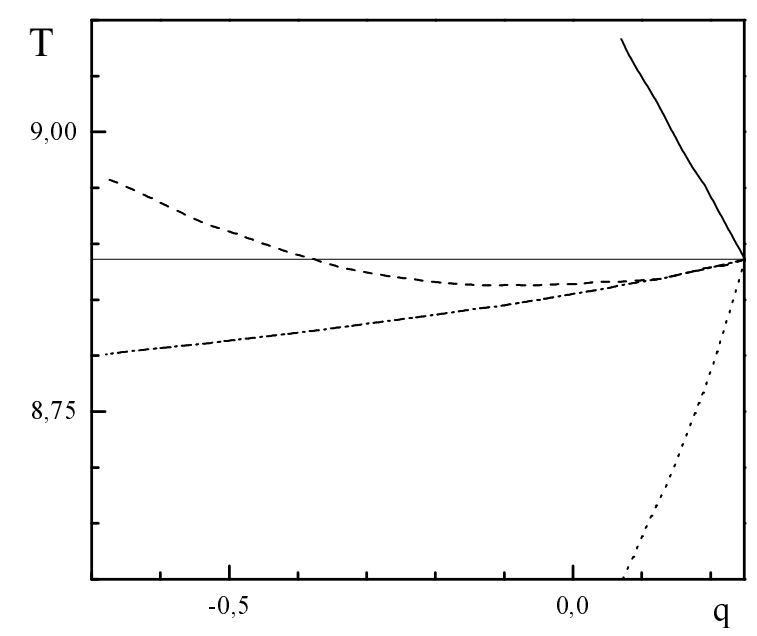

Fig. 2. The dependence of modulation period $T$ on the effective temperature $q$ in the model (1), (9). The solid curve shows $T(q)$ for the case $g=-1$, the dashed curve is for $g=+0.1$, the dotted curve is for $g=+1$. The dashed-dotted curve is calculated in the approach (3), $g=+0.1$. The solid line corresponds to the value $T(q)=2 \pi / b_{0} \approx 8.886$.

In the previous papers $[8,9]$ we have developed the approach to find the novel solutions of equation (2).

According to $[8,9]$, the solutions of equation (2) can satisfy the expression 


$$
\varphi^{\prime 2}(x)=\sum_{n=0}^{\infty} a_{n} \varphi^{2 n}
$$

The coefficients $a_{n}$ in the equation (5) are found from the infinite system of linking algebraic equations.

In general, the solving of equation (5) and the corresponding system for the coefficients $a_{n}$ is not a more simple task than the initial problem (1) $-(2)$. Nevertheless, the representation (5) allows to facilitate our consideration essentially. Really, we can account the truncated sum

$$
\varphi^{\prime 2}(x)=\sum_{n=0}^{N} a_{n} \varphi^{2 n}
$$

as the $N$-th approximation of the exact solution (5). In the case of $(6)$ the coefficients $a_{0}, a_{1}, \ldots, a_{N}$ can be found by the minimization of thermodynamical potential (1) in respect to them.

According to (6) the first approximation which gives the spatially periodic solutions corresponds to $N=1$ and the equation (6) has the form:

$$
\left(\varphi^{\prime}\right)^{2}=a_{0}+a_{1} \varphi^{2}
$$

It is easy to see that equation ( 7 ) is the differential equation for function (3), i.e. the case $N=1$ represents the one-harmonic approximation.

The simplest approximation which generates nonlinear distribution is the case of $N=2$ :

$$
\left(\varphi^{\prime}\right)^{2}=a_{0}+a_{1} \varphi^{2}+a_{2} \varphi^{4}
$$

Equation (8)is the differential equation for the Jacobi elliptic functions [12]. Among the Jacobi elliptic functions only the elliptic sinus $\operatorname{sn}(x, k)$, the elliptic cosine $\operatorname{cn}(x, k)$ and the function $\operatorname{dn}(x, k)$ are finite on the real $x$-axis. The elliptic modulus $k$ satisfies the inequality $0<k^{2}<1$.

The distribution (3) is an essentially linear one. At the same time, in the real physical systems the order parameter distribution is strictly linear only in the close vicinity of the point of transition from the disordered phase into the incommensurate one (more exactly, only at this point). The farther the system is from this point the larger the contribution of higher harmonics becomes. Hence, for the correct description of order parameter distribution the non-linear solutions are more appropriate.

Among the functions satisfying equation (8) the following one can be considered as the non-linear generalization of distribution (3) [9,12]:

$$
\varphi(x)=a \cdot \operatorname{sn}(b x, k)
$$

If the elliptic modulus is small $\left(k^{2} \approx 0\right)$ distribution $(9)$ is equivalent to the one-harmonic approximation (3). As $k$ approaches unity the contribution of higher harmon- ics grows. It should be noted that only odd harmonics $\sin [(2 n+1)] b x, n=0,1,2, \ldots$, are present in the Fourier series of distribution (9) which is in complete agreement with the symmetrical properties of equation (2) and the numerical analysis of the problem (1)-(2) [2]. In the limiting case $k^{2} \approx 1$ dependence $(9)$ corresponds to the periodic structure of kink-like spatial solitons.

As we have said above distribution (9) is the simplest non-linear approximation of the exact solution (5). To get closer to the exact solution we should use the approximations with $N \geq 3$. In this case the order parameter distribution is expressed through the hyperelliptic functions. But when we preserve the terms $\varphi^{2 n}, n \geq 3$, in expression (6) which can mean, in particular, that large values of the order parameter become important. In the Landau-type theory such a situation needs an additional detailed study.

It should be noted that there exists the unique set of values of parameters $a, b, k$ for which solution (9) is an exact one to equation (2) [9]. But this partial exact solution does not correspond to the absolute minimum of thermodynamical potential [9] and, consequently, it does not describe the equilibrium state which is under consideration in the current paper.

\section{NUMERICAL INVESTIGATION OF Sn-DISTRIBUTION}

In this section we represent the results of the investigation of properties of the approximate equilibrium distributions of one-component order parameter (9).

The variational equations for parameters $a, b, k$ of distribution (9) are complex expressions containing complete elliptic integrals of the first $K(k)$ and second $E(k)$ kind. Therefore we use a numerical procedure to find the equilibrium values of $a, b, k$.

The properties of system (1) depend on the current values of parameters $g, q, p$. Among these generalized material parameters the "temperature" $q$ and the magnitude $g$ of the interaction between $\varphi(x)$ and $\nabla \varphi(x)$ fields [9] are of main interest. So we assume that $p=1$ for the sake of simplicity.

In our calculations the effective temperature $q$ changes in the interval $q_{L}-\Delta q \leq q \leq q_{I}$. Here $q_{I}=\gamma^{2} / 4$ is the point where the disordered phase becomes absolutely unstable and the system undergoes the second order phase transition into the incommensurate phase [11], $q_{L}=q_{L}(g)$ is the point of first order phase transition into the commensurate phase, $\Delta q$ stands for some arbitrary small $q$-interval which is used to locate the point $q_{L}$ during the calculations.

The parameter $g$ changes in the interval $-10<g<$ $\sqrt{40 / 3}$. The lower limit is an arbitrary but large enough value needed to evaluate the tendency of system characteristics behaviour. As for the upper limit, it is the boundary value for the parameter $g$ (see Appendix).

Figs. 1-3 show the characteristic dependence of wave number $b$, modulation period $T$ and thermodynamical potential $\Phi$ on the effective temperature $q$ for different 
values of the parameter $g$. The dependencies of amplitude $a(q)$ and elliptic modulus $k(q)$ are monotonically increasing functions as the temperature is lowered.

The decrease of parameter $g$ from +1 to -10 leads to the following consequences.

a). The absolute value of $q_{L}$ becomes smaller $\left(q_{L}\right.$ is negative for all the values of $g$ ). In other words, the temperature interval of incommensurate phase existence decreases because the temperature $q_{I}$ does not depend on the $g$ value. For example, if $g=-1$ then $q_{L}=-0.69$, if $g=-10$ then $q_{L}=-0.23$.

b). The elliptic modulus value $k_{L}$ corresponding to the point $q_{L}$ increases: for $g=-1-k_{L}=0.53$, for $g=-10$ $-k_{L}=0.888$. It means that the contribution of higher harmonics in the order parameter distribution becomes larger as $g$ decreases.

c). The modulation period grows faster. But the following should be emphasized circumstances.

Firstly, there is no strong correlation between the modulation period and the wave number as it takes place for models (3), (4). For distribution (9), the period $T=4 K(k) / b[12]$ increases even when the parameter $b$ increases as well. As for the wave number itself, it begins to decrease as the temperature is lowered only for the large enough value of $|g|, g<0$.

Secondly, for some positive values of $g$, the modulation period begins to decrease. But there also exist such values of $g>0$ (for example, $g=0.1$ ) for which the period $T$ decreases in some vicinity of $q_{I}$ but then it starts to grow.

\section{DISCUSSION}

In the present paper we investigate some non-linear properties of the incommensurate state in the systems with one-component order parameter.

Our consideration is based on approach (6) which gives the "recipe" to obtain the solutions of variational equation with different accuracy. The considered solution (9) is one of the essentially non-linear approximations of the order parameter distribution in the incommensurate phase. The distribution (9) permits to describe the order parameter behaviour not only in the vicinity of the point of the disordered-incommensurate phase transition but near the transition from the incommensurate phase into the commensurate state as well. Using model (9) we express the order parameter dependence on the spatial coordinate through the well-known special function with three varying parameters rather than through the harmonic series which, in general, is infinite. This circumstance essentially simplifies the analysis of the non-linear properties of the systems with one-component order parameter.

The specific feature of the elliptic sinus is that it essentially differs from distribution (3) only in close vicinity of the point $k^{2}=1$ [12]. For the characteristic values of $k_{L}$ occurring in our calculations, the contribution of the second harmonic $a_{3} \cdot \sin (3 b x)$ in $(9)$ in respect to the basic harmonic (3) equals several percent [12]. That is in good agreement with the experimental data [1].

When the parameter $g$ is negative and $|g|$ is large enough the $k_{L}$ values close to unity can arise in the vicinity of the point of transition from the incommensurate phase into the commensurate state. It enables the appearance of distributions resembling the multi-soliton structures. But, at the same time, the growth of $g$ absolute value leads to a decrease in the incommensurate phase existence interval. Hence, even neglecting the fact that the large negative values of $g$ can never occur in real systems, we see that the possibility of the appearance of multi-soliton states can be in contradiction with the possibility to observe not only the non-linear distributions but the incommensurate phase in the experiment as well.

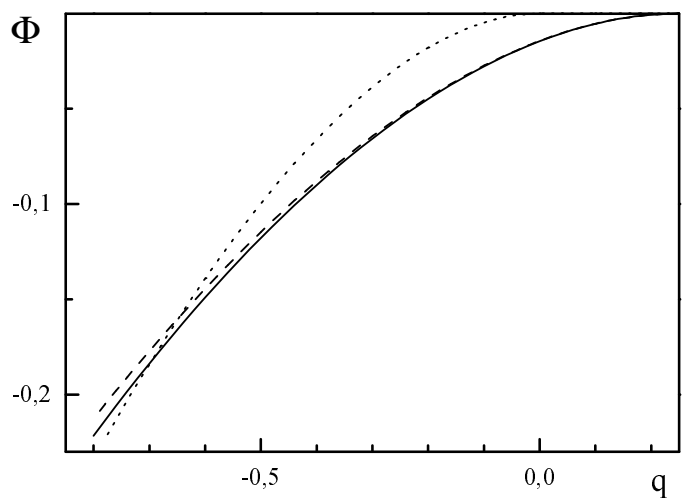

Fig. 3. The dependence of thermodynamical potential $\Phi$ on the effective temperature $q$. The parameter $g$ is equal to $g=-1$. The solid curve is for model (9), the dashed curve is for approach (3), the dotted curve shows the thermodynamical potential of commensurate phase.

Our results confirm that the $g$-term plays a very important role in the phenomenology of systems with onecomponent order parameter. But as it follows from what has been said above, in the case of model (9) the novel mechanism of the order parameter dependence on the external parameters exists.

This mechanism differs from the ordinary one (4) based on the $g$-term utilization. The essentially nonlinear character of solution (9) permits to take into account, in particular, the dependence on temperature even when $g=0$. Using the solution (9) we make the requirements on the parameter $g$ value weaker. This circumstance enhances the ability of theory to describe the properties of one-component order parameter systems. It is also important from the point of view that the equations analogous to equation (2) are encountered not only in the physical systems (ferroelectrics etc.) but in chemical, biological, computer systems as well [13] where the role of $g$-term can be very different.

Therefore, in comparison with the models in which the order parameter distribution is approximated with 
the harmonic series model (1), (9) allows to understand the non-linear behaviour of systems with the onecomponent order parameter in more detail and provides a new mechanism for the description of the dependence of system characteristics on the external parameters.

\section{ACKNOWLEDGMENTS}

The research described in this publication was supported, in part, by the State Fund of Fundamental Researches of Ukraine under Grant No. 2.4/691. S. V. Berezovsky is also indebted to the State Committee of Science and Technology of Ukraine, scholarships 235/96.

\section{APPENDIX. System global stability and $\varphi^{\prime 2} \varphi^{2}-$ term}

If $g<0$ then the contribution of the $\left(-g \cdot \varphi^{2} \varphi^{2}\right)-$ term in thermodynamical potential is always positive and can not destroy the global stability of system (1). But it can be broken when $g$ is positive [14]. Nevertheless, there exists the interval of parameter $g>0$ values for which the global stability of (1) (at least with respect to the considered distributions) is preserved.

To prove this statement we consider in the first place the limiting case of function (9) $k=0$. If $k=0$ then [12]

$$
\operatorname{sn}(b x, 0)=\sin (b x)
$$

and the expression for the thermodynamical potential has the form

$$
\begin{aligned}
\Phi & =\frac{1}{2} a^{2} b^{4}-\frac{1}{8} g a^{4} b^{2}-\frac{1}{2} \gamma a^{2} b^{2} \\
& +\frac{1}{2} q a^{2}+\frac{3}{16} p a^{4}+\frac{5}{48} a^{6} .
\end{aligned}
$$

Mathematically the global stability breaking means that the thermodynamical potential of the system approaches minus infinity $\Phi \rightarrow-\infty$ for some values of $a, b$. For the concrete expression (A2) such points are respectively $a=\infty$ and $b=\infty$ (we consider physically meaningful solutions so we assume that $a \geq 0$ and $b \geq 0$ ). When only one of the parameters $a, b$ becomes infinite the thermodynamical potential (A2) is always positive because the leading coefficients on $a$ and $b$ are positive. But when $a, b$ are of the same order and approach infinity and $g>0$ then the function (A2) can become negative. As long as $a$ and $b$ must change simultaneously we can assume that $b$ is a function on $a: b=f(a)$ and furthermore that $b=\sum_{n=0}^{\infty} c_{n} a^{n}$. It is simple to see that the leading coefficient in $a$-terms can be negative only for the case $b=c \cdot a$. Indeed, when $b \sim a^{m}, m \geq 2$, the term in $a^{2} b^{4} \sim a^{2+4 m}$, where $2+4 m>6$, is the leading one and it is always positive. For the case of the correlation $b=c \cdot a$ function (A2) transforms into

$$
\begin{gathered}
\Phi=A \cdot a^{6}+\left(\frac{3}{16} p-\frac{1}{2} \gamma c^{2}\right) \cdot a^{4}+\frac{1}{2} q a^{2}, \\
A=\frac{1}{2} c^{4}-\frac{1}{8} g c^{2}+\frac{5}{48} .
\end{gathered}
$$

If for the current value of $g$ and for some real values of $c$ coefficient $A$ can become negative then the system thermodynamical potential is globally unstable. In other words, to provide the thermodynamical potential global stability function $A(c)$ must be always positive, i.e. polynomial (A4) must not have real roots. This requirement leads to the inequality

$$
g<\sqrt{\frac{40}{3}} \approx 3.65
$$

When $k^{2}>0$ the restriction (A5) becomes less strict. Really, for $k^{2} \rightarrow 1$ the thermodynamical potential (1) can be written in the form

$$
\begin{aligned}
\frac{\Phi}{L} & \approx a^{2}\left\{q\left(1+\frac{1}{2} k^{2}\right)-\Lambda^{-1}\left(q+\frac{2}{3} \gamma b^{2}-\frac{8}{15} b^{4}\right)\right\} \\
& +a^{4}\left\{\frac{p}{2}\left(1+k^{\prime 2}\right)-\Lambda^{-1}\left(\frac{2}{3} p+\frac{2}{15} g b^{2}\right)\right\} \\
& +\frac{1}{3} a^{6}\left(1-\frac{23}{15} \Lambda^{-1}\right) .
\end{aligned}
$$

Repeating the foregoing consideration we come to the conclusion that $g$ must satisfy the following condition

$$
\begin{aligned}
& g<\sqrt{40 \Lambda-\frac{184}{3}}, \\
& \Lambda=\ln \frac{4}{\sqrt{1-k^{2}}} .
\end{aligned}
$$

If $k^{2}=0.9$ then $\Lambda=2.54$ and $g<6.3$. When $k^{2} \rightarrow 1$ then $\Lambda \rightarrow \infty$. In the limit $k^{2}=1$ there is no any restriction on the value of parameter $g$ if we keep in mind the system's global stability.

Therefore, if we assume that the order parameter distribution has the form of $(9), 0 \leq k^{2} \leq 1$, then system (1) is globally stable not only for the negative values of parameter $g$ but for the interval $0 \leq g<\sqrt{40 / 3}$ as well. 
[1] H. Z. Cummins, Phys. Rep. 185, 211 (1990).

[2] A. D. Bruce, R. A. Cowley, A. F. Murrey, J. Phys. C 11, 3591 (1978).

[3] J. C. Tolédano, P. Tolédano, The Landau Theory of Phase Transitions (World Scientific Publ. Co., Singapore, 1987).

[4] Y. Ishibashi, H. Shiba, J. Phys. Soc. Jpn. 45, 409 (1978).

[5] S. L. Qiu, Mitra Dutta, H. Z. Cummins, J. P. Wicksted, S. M. Shapiro, Phys. Rev. B 34, 7901 (1986).

[6] M. R. Chaves, A. Almeida, J. M. Kiat, J. C. Tolédano, J. Schneck, R. Glass, W. Schwarz, J. L. Ribeiro, A. Klöpperpieper, J. Albers, Phys. Status Solidi B 189, 97 (1995).

[7] A. P. Levanyuk, D. G. Sannikov, Fiz. Tverd. Tela 18,
423 (1976); Sov. Phys. — Solid State 18, 245 (1976).

[8] V. F. Klepikov, S. V. Berezovsky, Cond. Matt. Phys. 8, 69 (1996).

[9] V. F. Klepikov, A. I. Olemskoi, S. V. Berezovsky, Metallofiz. nov. tekhnol. 19, 32 (1997).

[10] V. F. Klepikov, Sov. Phys. - Low Temp. Phys. 17, 1166 (1991).

[11] A. Michelson, Phys. Rev. B 16, 577 (1977).

[12] Handbook of Mathematical Functions, edited by A. Abramowitz, I. A. Stegun (Dover, New York, 1972).

[13] M. C. Cross, P. C. Hohenberg, Rev. Mod. Phys. 65, 851 (1993).

[14] S. B. Goryachev, Phys. Rev. Lett. 72, 1850 (1994).

\title{
НЕЛ ІНІЙНІ ВЛ АСТИВОСТІ НЕСУМІРНИХ ФАЗ У СИСТЕМАХ ЗІ СКАЛЯРНИМ ПАРАМЕТРОМ ПОРЯДКУ
}

\author{
С. В. Березовський, В. Ф. Клепіков, Ю. В. Середа \\ Наукобо-технічний центр електрофізичной обробки \\ Начіональноӥакадемӥ наук Украӥни \\ вул. Чернишевського, 28, а/с 8812, Харків, 310002, Украӥна, \\ meл. (0572) 40-42-82,e-mail: ipct@pem.kharkov.ua
}

\begin{abstract}
Розглянуто нелінійні властивості розподілів параметра порядку в системах із однокомпонентним параметром порядку. Показано, що розв'язки, які вира жаються через еліптичні функції Якобі, дають більше інформації про поведінку таких систем порівняно з моделями, де розподіл параметра порядку набли жений гармонічним рядом. Розроблений підхід забезпечує новий механізм зале жности характеристик системи від зовнішніх параметрів.
\end{abstract}

